\title{
BOUNDEDNESS IN THE PERTURBED FUNCTIONAL DIFFERENTIAL SYSTEMS
}

\author{
Dong Man Im*, Sang Il Chol**, and Yoon Hoe Goo***
}

ABSTRACT. In this paper, we investigate bounds for solutions of the the perturbed functional differential systems.

\section{Introduction}

As is traditional in a pertubation theory of nonlinear differential systems, the behavior of solutions of a perturbed system is determined in terms of the behavior of solutions of an unperturbed system. Among useful methods for investigating the qualitative behavior of the solutions of perturbed nonlinear system of differential systems, there are the method of variation of constants formula, Lyapunov' second method, and the use of inequalities. The theory of integral inequalities can be employed to study various properties of nonlinear differential systems. In the presence the method of integral inequalities is as efficient as the direct Lyapunov's method.

Pinto $[13,14]$ introduced $h$-stability(hS) which is an important extention of the notions of exponential asymptotic stability and uniform Lipschitz stability. Also, he obtained some properties about asymptotic behavior of solutions of perturbed $h$-systems, some general results about asymptotic integration and gave some important examples in [14]. He obtained a general variational $h$-stability and some properties about asymptotic behavior of solutions of differential systems called $h$-systems. Also, he studied some general results about asymptotic integration and gave some important examples in [13]. Choi and Ryu [3], Choi, Koo[5], and Choi et al. [4] investigated bounds of solutions for the perturbed

Received May 22, 2014; Accepted July 18, 2014.

2010 Mathematics Subject Classification: Primary 34D10.

Key words and phrases: $h$-stability, $t_{\infty}$-similarity, nonlinear nonautonomous system.

Correspondence should be addressed to Yoon Hoe Goo, yhgoo@hanseo.ac.kr. 
functional differential systems. Also, Goo $[7,8,9,10]$ studied the boundedness of solutions for the perturbed functional differential systems.

In this paper, we investigate bounds of solutions of the perturbed functional differential systems.

\section{Preliminaries}

We consider the perturbed functional differential equation

$$
y^{\prime}=f(t, y)+\int_{t_{0}}^{t} g(s, y(s), T y(s)) d s, y\left(t_{0}\right)=y_{0},
$$

where $t \in \mathbb{R}^{+}=[0, \infty), x \in \mathbb{R}^{n}, f \in C\left(\mathbb{R}^{+} \times \mathbb{R}^{n}, \mathbb{R}^{n}\right), f(t, 0)=0$, the derivative $f_{x} \in C\left(\mathbb{R}^{+} \times \mathbb{R}^{n}, \mathbb{R}^{n}\right), g \in C\left(\mathbb{R}^{+} \times \mathbb{R}^{n}, \mathbb{R}^{n}\right), g(t, 0,0)=0$ and $T$ is a continuous operator mapping from $C\left(\mathbb{R}^{+}, \mathbb{R}^{n}\right)$ into $C\left(\mathbb{R}^{+}, \mathbb{R}^{n}\right)$. The symbol $|\cdot|$ will be used to denote arbitrary vector norm in $\mathbb{R}^{n}$. We assume that for any two continuous functions $u, v \in C(I)$ where $I$ is the closed interval, the operator $T$ satisfies the following property:

$$
u(t) \leq v(t), 0 \leq t \leq t_{1}, t_{1} \in I,
$$

implies $T u(t) \leq T v(t), 0 \leq t \leq t_{1}$, and $|T u| \leq T|u|$.

Equation (2.1) can be considered as the perturbed equation of

$$
x^{\prime}(t)=f(t, x(t)), \quad x\left(t_{0}\right)=x_{0},
$$

Let $x\left(t, t_{0}, x_{0}\right)$ be denoted by the unique solution of (2.2) passing through the point $\left(t_{0}, x_{0}\right) \in \mathbb{R}^{+} \times \mathbb{R}^{n}$ such that $x\left(t_{0}, t_{0}, x_{0}\right)=x_{0}$. Also, we can consider the associated variational systems around the zero solution of (2.2) and around $x(t)$, respectively,

$$
v^{\prime}(t)=f_{x}(t, 0) v(t), v\left(t_{0}\right)=v_{0}
$$

and

$$
z^{\prime}(t)=f_{x}\left(t, x\left(t, t_{0}, x_{0}\right)\right) z(t), z\left(t_{0}\right)=z_{0} .
$$

The fundamental matrix $\Phi\left(t, t_{0}, x_{0}\right)$ of (4) is given by

$$
\Phi\left(t, t_{0}, x_{0}\right)=\frac{\partial}{\partial x_{0}} x\left(t, t_{0}, x_{0}\right),
$$

and $\Phi\left(t, t_{0}, 0\right)$ is the fundamental matrix of $(2.3)$.

We recall some notions of $h$-stability [13]. 
Definition 2.1. The system (2.2) (the zero solution $x=0$ of $(2.2)$ ) is called an $h$-system if there exist a constant $c \geq 1$ and a positive continuous function $h$ on $\mathbb{R}^{+}$such that

$$
|x(t)| \leq c\left|x_{0}\right| h(t) h\left(t_{0}\right)^{-1}
$$

for $t \geq t_{0} \geq 0$ and $\left|x_{0}\right|$ small enough (here $h(t)^{-1}=\frac{1}{h(t)}$ ).

Definition 2.2. The system (2.2) (the zero solution $x=0$ of $(2.2)$ ) is called (hS) $h$-stable if there exist $\delta>0$ such that (2.2) is an $h$-system for $\left|x_{0}\right| \leq \delta$ and $h$ is bounded.

Let $\mathcal{M}$ denote the set of all $n \times n$ continuous matrices $A(t)$ defined on $\mathbb{R}^{+}$and $\mathcal{N}$ be the subset of $\mathcal{M}$ consisting of those nonsingular matrices $S(t)$ that are of class $C^{1}$ with the property that $S(t)$ and $S^{-1}(t)$ are bounded. The notion of $t_{\infty}$-similarity in $\mathcal{M}$ was introduced by Conti [6].

Definition 2.3. A matrix $A(t) \in \mathcal{M}$ is $t_{\infty}$-similar to a matrix $B(t) \in$ $\mathcal{M}$ if there exists an $n \times n$ matrix $F(t)$ absolutely integrable over $\mathbb{R}^{+}$, i.e.,

such that

$$
\int_{0}^{\infty}|F(t)| d t<\infty
$$

$$
\dot{S}(t)+S(t) B(t)-A(t) S(t)=F(t), \cdot=\frac{d}{d t}
$$

for some $S(t) \in \mathcal{N}$.

We give some related properties that we need in the sequal.

Lemma 2.4. [14] The linear system

$$
x^{\prime}=A(t) x, x\left(t_{0}\right)=x_{0},
$$

where $A(t)$ is an $n \times n$ continuous matrix, is an $h$-system (respectively $h$-stable) if and only if there exist $c \geq 1$ and a positive and continuous (respectively bounded) function $h$ defined on $\mathbb{R}^{+}$such that

$$
\left|\phi\left(t, t_{0}\right)\right| \leq c h(t) h\left(t_{0}\right)^{-1}
$$

for $t \geq t_{0} \geq 0$, where $\phi\left(t, t_{0}\right)$ is a fundamental matrix of (2.6).

We need Alekseev formula to compare between the solutions of (2.2) and the solutions of perturbed nonlinear system

$$
y^{\prime}=f(t, y)+g(t, y), y\left(t_{0}\right)=y_{0},
$$


where $g \in C\left(\mathbb{R}^{+} \times \mathbb{R}^{n}, \mathbb{R}^{n}\right)$ and $g(t, 0)=0$. Let $y(t)=y\left(t, t_{0}, y_{0}\right)$ denote the solution of (2.8) passing through the point $\left(t_{0}, y_{0}\right)$ in $\mathbb{R}^{+} \times \mathbb{R}^{n}$.

The following is a generalization to nonlinear system of the variation of constants formula due to Alekseev [1].

Lemma 2.5. If $y_{0} \in \mathbb{R}^{n}$, then for all $t$ such that $x\left(t, t_{0}, y_{0}\right) \in \mathbb{R}^{n}$,

$$
y\left(t, t_{0}, y_{0}\right)=x\left(t, t_{0}, y_{0}\right)+\int_{t_{0}}^{t} \Phi(t, s, y(s)) g(s, y(s)) d s .
$$

TheOREM 2.6. [3] If the zero solution of (2.2) is $h S$, then the zero solution of (2.3) is $h S$.

Theorem 2.7. [4] Suppose that $f_{x}(t, 0)$ is $t_{\infty}$-similar to $f_{x}\left(t, x\left(t, t_{0}, x_{0}\right)\right)$ for $t \geq t_{0} \geq 0$ and $\left|x_{0}\right| \leq \delta$ for some constant $\delta>0$. If the solution $v=0$ of (2.3) is hS, then the solution $z=0$ of (2.4) is $h S$.

Lemma 2.8. [7] Let $u, \lambda_{1}, \lambda_{2}, \lambda_{3} \in C\left(\mathbb{R}^{+}\right), w \in C((0, \infty))$, and $w(u)$ be nondecreasing in $u, u \leq w(u)$. Suppose that for some $c>0$,

$u(t) \leq c+\int_{t_{0}}^{t} \lambda_{1}(s) w(u(s)) d s+\int_{t_{0}}^{t} \lambda_{2}(s)\left(\int_{t_{0}}^{s} \lambda_{3}(\tau) u(\tau) d \tau\right) d s, 0 \leq t_{0} \leq t$.

Then

$$
u(t) \leq W^{-1}\left[W(c)+\int_{t_{0}}^{t}\left(\lambda_{1}(s)+\lambda_{2}(s) \int_{t_{0}}^{s} \lambda_{3}(\tau) d \tau\right) d s\right], t_{0} \leq t<b_{1},
$$

where $W(u)=\int_{u_{0}}^{u} \frac{d s}{w(s)}, W^{-1}(u)$ is the inverse of $W(u)$, and

$b_{1}=\sup \left\{t \geq t_{0}: W(c)+\int_{t_{0}}^{t}\left(\lambda_{1}(s)+\lambda_{2}(s) \int_{t_{0}}^{s} \lambda_{3}(\tau) d \tau\right) d s \in \operatorname{domW}^{-1}\right\}$.

Lemma 2.9. [7] Let $u, p, q, w, r \in C\left(\mathbb{R}^{+}\right), w \in C((0, \infty))$, and $w(u)$ be nondecreasing in $u, u \leq w(u)$. Suppose that for some $c>0$,

$u(t) \leq c+\int_{t_{0}}^{t}\left(p(s) \int_{t_{0}}^{s}\left(q(\tau) w(u(\tau))+v(\tau) \int_{t_{0}}^{\tau} r(a) u(a) d a\right) d \tau\right) d s, t \geq t_{0}$.

Then

$u(t) \leq W^{-1}\left[W(c)+\int_{t_{0}}^{t}\left(p(s) \int_{t_{0}}^{s}\left(q(\tau)+v(\tau) \int_{t_{0}}^{\tau} r(a) d a\right) d \tau\right) d s\right], t_{0} \leq t<b_{1}$,

where $W$ and $W^{-1}$ are the same functions as in Lemma 2.8, and

$$
\begin{gathered}
b_{1}=\sup \left\{t \geq t_{0}: W(c)+\int_{t_{0}}^{t}\left(p(s) \int_{t_{0}}^{s}\left(q(\tau)+v(\tau) \int_{t_{0}}^{\tau} r(a) d a\right) d \tau\right) d s\right. \\
\left.\in \text { domW }^{-1}\right\} .
\end{gathered}
$$




\section{Main results}

In this section, we investigate the bounded property for the nonlinear functional differential systems.

Theorem 3.1. Let $a, b, k, u, w \in C\left(\mathbb{R}^{+}\right), w(u)$ be nondecreasing in $u, u \leq w(u)$, and $\frac{1}{v} w(u) \leq w\left(\frac{u}{v}\right)$ for some $v>0$. Suppose that $f_{x}(t, 0)$ is $t_{\infty}$-similar to $f_{x}\left(t, x\left(t, t_{0}, x_{0}\right)\right)$ for $t \geq t_{0} \geq 0$ and $\left|x_{0}\right| \leq \delta$ for some constant $\delta>0$, the solution $x=0$ of (2.2) is $h S$ with the nondecreasing function $h$, and $g$ in (2.1) satisfies

$$
\left|\int_{t_{0}}^{s} g(\tau, y(\tau), T y(\tau)) d \tau\right| \leq a(s) w(|y(s)|+b(s)|T y(s)|), t \geq t_{0} \geq 0,
$$

and

$$
|T y| \leq \int_{t_{0}}^{t} k(s)|y(s)| d s,
$$

where $\int_{t_{0}}^{\infty} a(s) d s<\infty, \int_{t_{0}}^{\infty} b(s) d s<\infty$, and $\int_{t_{0}}^{\infty} k(s) d s<\infty$. Then, any solution $y(t)=y\left(t, t_{0}, y_{0}\right)$ of $(2.1)$ is bounded on $\left[t_{0}, \infty\right)$ and it satisfies

$$
|y(t)| \leq h(t) W^{-1}\left[W(c)+c_{2} \int_{t_{0}}^{t}\left(a(s)+b(s) \int_{t_{0}}^{s} k(\tau) d \tau\right) d s\right], t_{0} \leq t<b_{1}
$$

where $c$ is a positive constant, $W$ and $W^{-1}$ are the same functions as in Lemma 2.8, and

$$
b_{1}=\sup \left\{t \geq t_{0}: W(c)+c_{2} \int_{t_{0}}^{t}\left(a(s)+b(s) \int_{t_{0}}^{s} k(\tau) d \tau\right) d s \in \operatorname{dom}^{-1}\right\} .
$$

Proof. Let $x(t)=x\left(t, t_{0}, y_{0}\right)$ and $y(t)=y\left(t, t_{0}, y_{0}\right)$ be solutions of (2.2) and (2.1), respectively. By Theorem 2.6, since the solution $x=0$ of $(2.2)$ is $\mathrm{hS}$, the solution $v=0$ of $(2.3)$ is $\mathrm{hS}$. Therefore, by Theorem 2.7, the solution $z=0$ of (2.4) is hS. By Lemma 2.4, Lemma 2.5, and the nondecreasing property of the function $h$, we have

$$
\begin{aligned}
|y(t)| \leq & |x(t)|+\int_{t_{0}}^{t}|\Phi(t, s, y(s))| \int_{t_{0}}^{s} g(\tau, y(\tau), T y(\tau)) d \tau \mid d s \\
\leq & c_{1}\left|y_{0}\right| h(t) h\left(t_{0}\right)^{-1}+\int_{t_{0}}^{t} c_{2} h(t) a(s) w\left(\frac{|y(s)|}{h(s)}\right) d s \\
& +\int_{t_{0}}^{t} c_{2} h(t) b(s) \int_{t_{0}}^{s} k(\tau) \frac{|y(\tau)|}{h(\tau)} d \tau d s .
\end{aligned}
$$


Set $u(t)=|y(t)| h(t)^{-1}$. Then, by Lemma 2.8, we obtain $|y(t)| \leq h(t) W^{-1}\left[W(c)+c_{2} \int_{t_{0}}^{t}\left(a(s)+b(s) \int_{t_{0}}^{s} k(\tau) d \tau\right) d s\right], t_{0} \leq t<b_{1}$, where $c=c_{1}\left|y_{0}\right| h\left(t_{0}\right)^{-1}$. This completes the proof.

REMARK 3.2. Letting $k(\tau)=0$ in Theorem 3.1, we have the similar result as that of Theorem 3.2 in [8].

Theorem 3.3. Let $a, b, k, u, w \in C\left(\mathbb{R}^{+}\right), w(u)$ be nondecreasing in $u$, $u \leq w(u)$, and $\frac{1}{v} w(u) \leq w\left(\frac{u}{v}\right)$ for some $v>0$. Suppose that the solution $x=0$ of (2.2) is $h S$ with a nondecreasing function $h$ and the perturbed term $g$ in (2.1) satisfies

$$
|\Phi(t, s, y) g(t, y, T y)| \leq a(s) w(|y|)+b(s)|T y|, t \geq t_{0} \geq 0,
$$

and

$$
|T y| \leq \int_{t_{0}}^{t} k(s)|y(s)| d s,
$$

where $\int_{t_{0}}^{\infty} a(s) d s<\infty, \int_{t_{0}}^{\infty} b(s) d s<\infty$, and $\int_{t_{0}}^{\infty} k(s) d s<\infty$. Then any solution $y(t)=y\left(t, t_{0}, y_{0}\right)$ of $(2.1)$ is bounded on $\left[t_{0}, \infty\right)$ and it satisfies

$$
|y(t)| \leq h(t) W^{-1}\left[W(c)+\int_{t_{0}}^{t}\left(a(s)+b(s) \int_{t_{0}}^{s} k(\tau) d \tau\right) d s\right], t_{0} \leq t<b_{1} .
$$

where $W$ and $W^{-1}$ are the same functions as in Lemma 2.8, $\mathrm{c}$ is a positive constant, and

$$
b_{1}=\sup \left\{t \geq t_{0}: W(c)+\int_{t_{0}}^{t}\left(a(s)+b(s) \int_{t_{0}}^{s} k(\tau) d \tau\right) d s \in \operatorname{domW}^{-1}\right\} .
$$

Proof. Let $x(t)=x\left(t, t_{0}, y_{0}\right)$ and $y(t)=y\left(t, t_{0}, y_{0}\right)$ be solutions of (2.2) and (2.1), respectively. By Lemma 2.5, we obtain

$$
\begin{aligned}
|y(t)| \leq & |x(t)|+\int_{t_{0}}^{t}|\Phi(t, s, y(s)) g(s, y(s), T y(s))| d s \\
\leq & c_{1}\left|y_{0}\right| h(t) h\left(t_{0}\right)^{-1}+\int_{t_{0}}^{t}\left(a(s) w(|y(s)|)+b(s) \int_{t_{0}}^{s} k(\tau)|y(\tau)| d \tau\right) d s \\
\leq & c_{1}\left|y_{0}\right| h(t) h\left(t_{0}\right)^{-1}+\int_{t_{0}}^{t} a(s) h(t) w\left(\frac{|y(s)|}{h(s)}\right) d s \\
& +\int_{t_{0}}^{t} b(s) \int_{t_{0}}^{s} h(t) k(\tau) \frac{|y(\tau)|}{h(\tau)} d \tau d s
\end{aligned}
$$


since $h$ is nondecreasing. Set $u(t)=|y(t)| h(t)^{-1}$. Then, by Lemma 2.8, we have

$|y(t)| \leq h(t) W^{-1}\left[W(c)+\int_{t_{0}}^{t}\left(a(s)+b(s) \int_{t_{0}}^{s} k(\tau) d \tau\right) d s\right], t_{0} \leq t<b_{1}$,

where $c=c_{1}\left|y_{0}\right| h\left(t_{0}\right)^{-1}$. Therefore, we obtain the result.

REMARK 3.4. Letting $k(\tau)=0$ in Theorem 3.3, we have the similar result as that of Theorem 3.1 in [8].

TheOREM 3.5. Let $a, b, k, u, w \in C\left(\mathbb{R}^{+}\right), w(u)$ be nondeacreasing in $u, u \leq w(u)$, and $\frac{1}{v} w(u) \leq w\left(\frac{u}{v}\right)$ for some $v>0$. Suppose that $f_{x}(t, 0)$ is $t_{\infty}$-similar to $f_{x}\left(t, x\left(t, t_{0}, x_{0}\right)\right)$ for $t \geq t_{0} \geq 0$ and $\left|x_{0}\right| \leq \delta$ for some constant $\delta>0$. If the solution $x=0$ of (2.2) is an h-system with a positive continuous function $h$ and $g$ in (2.1) satisfies

$$
|g(t, y, T y)| \leq a(t) w(|y(t)|)+b(t)|T y(t)|, t \geq t_{0}, y \in \mathbb{R}^{n}
$$

and

$$
|T y(t)| \leq \int_{t_{0}}^{t} k(s)|y(s)| d s,
$$

where $a: \mathbb{R}^{+} \rightarrow \mathbb{R}^{+}$is continuous with

$$
\int_{t_{0}}^{\infty} \frac{1}{h(s)} \int_{t_{0}}^{s}\left(a(\tau) h(\tau)+b(\tau) \int_{t_{0}}^{\tau} h(r) k(r) d r\right) d \tau d s<\infty
$$

for all $t_{0} \geq 0, \int_{t_{0}}^{\infty} a(s) d s<\infty, \int_{t_{0}}^{\infty} b(s) d s<\infty$, and $\int_{t_{0}}^{\infty} k(s) d s<\infty$, then any solution $y(t)=y\left(t, t_{0}, y_{0}\right)$ of (2.1) satisfies

$$
|y(t)| \leq h(t) W^{-1}\left[W(c)+\int_{t_{0}}^{t} \frac{c_{2}}{h(s)} \int_{t_{0}}^{s}\left(a(\tau) h(\tau)+b(\tau) \int_{t_{0}}^{\tau} h(r) k(r) d r\right) d \tau d s\right],
$$

$t_{0} \leq t<b_{1}$, where $W$ and $W^{-1}$ are the same functions as in Lemma 2.8, $c$ is a positive constant, and

$$
\begin{aligned}
b_{1}=\sup \left\{t \geq t_{0}: W(c)\right. & +\int_{t_{0}}^{t} \frac{c_{2}}{h(s)} \int_{t_{0}}^{s}(a(\tau) h(\tau) \\
& \left.\left.+b(\tau) \int_{t_{0}}^{\tau} h(r) k(r) d r\right) d \tau d s \in \mathrm{domW}^{-1}\right\} .
\end{aligned}
$$

Proof. Let $x(t)=x\left(t, t_{0}, y_{0}\right)$ and $y(t)=y\left(t, t_{0}, y_{0}\right)$ be solutions of (2.2) and (2.1), respectively. By Theorem 2.6, since the solution $x=0$ of $(2.2)$ is $\mathrm{hS}$, the solution $v=0$ of $(2.3)$ is $\mathrm{hS}$. Therefore, by Theorem 
2.7, the solution $z=0$ of (2.4) is hS. By Lemma 2.4 and Lemma 2.5, we have

$$
\begin{aligned}
|y(t)| \leq & |x(t)|+\int_{t_{0}}^{t}|\Phi(t, s, y(s))| \int_{t_{0}}^{s}|g(\tau, y(\tau), T y(\tau))| d \tau d s \\
\leq & c_{1}\left|y_{0}\right| h(t) h\left(t_{0}\right)^{-1}+\int_{t_{0}}^{t} c_{2} \frac{h(t)}{h(s)} \int_{t_{0}}^{s} a(\tau) h(\tau) w\left(\frac{|y(\tau)|}{h(\tau)}\right) d \tau d s \\
& +\int_{t_{0}}^{t} c_{2} \frac{h(t)}{h(s)} \int_{t_{0}}^{s} b(\tau) \int_{t_{0}}^{\tau} h(r) k(r) \frac{|y(r)|}{h(r)} d r d \tau d s .
\end{aligned}
$$

Setting $u(t)=|y(t)| h(t)^{-1}$ and using Lemma 2.9, we obtain

$$
\begin{aligned}
|y(t)| \leq h(t) W^{-1}[W(c) & +\int_{t_{0}}^{t} \frac{c_{2}}{h(s)} \int_{t_{0}}^{s}(a(\tau) h(\tau) \\
& \left.\left.+b(\tau) \int_{t_{0}}^{\tau} h(r) k(r) d r\right) d \tau d s\right],
\end{aligned}
$$

$t_{0} \leq t<b_{1}$, where $c=c_{1}\left|y_{0}\right| h\left(t_{0}\right)^{-1}$. Hence, the proof is complete.

REMARK 3.6. Letting $k(\tau)=0$ in Theorem 3.5, we have the similar result as that of Theorem 3.5 in [8].

\section{Acknowledgment.}

The author is very grateful for the referee's valuable comments.

\section{References}

[1] V. M. Alekseev, An estimate for the perturbations of the solutions of ordinary differential equations, Vestn. Mosk. Univ. Ser. I. Math. Mech. 2 (1961), 28-36.

[2] S. K. Choi and N. J. Koo, h-stability for nonlinear perturbed systems, Ann. of Diff. Eqs. 11 (1995), 1-9.

[3] S. K. Choi and H. S. Ryu, h-stability in differential systems, Bull. Inst. Math. Acad. Sinica 21 (1993), 245-262.

[4] S. K. Choi, N. J. Koo, and H. S. Ryu, h-stability of differential systems via $t_{\infty}$-similarity, Bull. Korean. Math. Soc. 34 (1997), 371-383.

[5] S. K. Choi, N. J. Koo, and S. M. Song, Lipschitz stability for nonlinear functional differential systems, Far East J. Math. Sci(FJMS)I 5 (1999), 689-708.

[6] R. Conti, Sulla $t_{\infty}$-similitudine tra matricie l'equivalenza asintotica dei sistemi differenziali lineari, Rivista di Mat. Univ. Parma 8 (1957), 43-47.

[7] Y. H. Goo, Boundedness in the perturbed differential systems, J. Korean Soc. Math.Educ. Ser. B: Pure Appl. Math. 20 (2013), 223-232.

[8] Y. H. Goo, D. G. Park, and D. H. Ryu, Boundedness in perturbed differential systems, J. Appl. Math. and Informatics 30 (2012), 279-287. 
[9] Y. H. Goo, Boundedness in perturbed nonlinear differential systems, J. Chungcheong Math. Soc. 26 (2013), 605-613.

[10] Y. H. Goo, Boundedness in the perturbed nonlinear differential systems, Far East J. Math. Sci(FJMS) 79 (2013), 205-217.

[11] G. A. Hewer, Stability properties of the equation by $t_{\infty}$-similarity, J. Math. Anal. Appl. 41 (1973), 336-344.

[12] V. Lakshmikantham and S. Leela, Differential and Integral Inequalities: Theory and Applications Vol. I, Academic Press, New York and London, 1969.

[13] M. Pinto, Perturbations of asymptotically stable differential systems, Analysis 4 (1984), 161-175.

[14] M. Pinto, Stability of nonlinear differential systems, Applicable Analysis 43 (1992), 1-20.

Department of Mathematics Education

Cheongju University

Cheongju 360-764, Republic of Korea

E-mail: dmim@cheongju.ac.kr

Department of Mathematics

Hanseo University

Seosan 356-706, Republic of Korea

E-mail: schoi@hanseo.ac.kr

$* * *$

Department of Mathematics

Hanseo University

Seosan 356-706, Republic of Korea

E-mail: yhgoo@hanseo.ac.kr 